\title{
Product-Specific Regulatory Pathways to Approve Generic Drugs: The Need for Follow-up Studies to Ensure Safety and Effectiveness
}

\author{
Aaron S. Kesselheim ${ }^{1}$ Joshua J. Gagne ${ }^{1}$
}

Published online: 24 June 2015

(c) Springer International Publishing Switzerland 2015

\begin{abstract}
Generic drugs possessing the same active ingredients, dosage form, strength, route of administration, and labeling can be approved by the US Food and Drug Administration (FDA) as interchangeable with a brandname drug without needing to repeat the formal Phase I, II, and III clinical trials conducted by the original manufacturers. In recent years, the FDA has approved several generic drugs using product-specific testing to determine therapeutic equivalence in accordance with the unique features of the particular drug. These have been used in two primary situations: (1) cases for which certain bioequivalence studies were not relevant; and (2) cases of complex molecules that may require specially tailored pharmaceutical equivalence studies. Examples include venlafaxine extended release, acarbose, vancomycin capsules, sodium ferric gluconate, salmon calcitonin nasal spray, and enoxaparin. Product-specific approaches to demonstrating therapeutic equivalence are essential to avoid delays in low-cost generic drug availability but can have important clinical implications; yet, currently, there is no formal process in place to monitor the safety and effectiveness of generic drugs approved using modified regulatory pathways. Several strategies can be used to monitor the safety and effectiveness of generic drugs approved via productspecific determinations of therapeutic equivalence.
\end{abstract}

Joshua J. Gagne

jgagne1@partners.org

1 Program on Regulation, Therapeutics, and Law (PORTAL), Division of Pharmacoepidemiology and Pharmacoeconomics, Department of Medicine, Brigham and Women's Hospital and Harvard Medical School, 1620 Tremont Street, Suite 3030, Boston, MA 02120, USA

\section{Key Points}

Generic drugs are an essential component of the healthcare marketplace, although some physicians and patients remain skeptical about their safety and effectiveness.

The US Food and Drug Administration uses productspecific pathways to demonstrate therapeutic equivalence for some generic drugs, which can generate controversy.

Though it is important for the Food and Drug Administration to exercise flexibility in the tests it requires to permit drugs to demonstrate therapeutic equivalence, following up generic drug approvals using product-specific pathways with active surveillance studies would be useful in promoting widespread appropriate use of low-cost generic drugs.

\section{Introduction}

It has been 30 years since the US Congress revolutionized the prescription drug industry by passing the Drug Price Competition and Patent Term Restoration Act of 1984 (the Hatch-Waxman Act). One main goal of the legislation was to expedite regulatory approval of generic prescription drugs-lower cost but therapeutically equivalent versions of brand-name products - by allowing them to be approved as safe and effective by the US Food and Drug Administration (FDA) if they were therapeutically equivalent, i.e., bioequivalent as well as pharmaceutically equivalent. 
These concepts allow generic drugs possessing the same active ingredients, dosage form, strength, route of administration, and labeling to meet the FDA's approval standards based on in vitro and in vivo testing without needing to repeat the formal Phase I, II, and III clinical trials conducted by the original brand-name manufacturers. Avoiding repetitive high-cost clinical trials for what ischemically and biologically - the same drug allows generic manufacturers to market inexpensive versions of brandname products after expiration of the brand-name version's patent and exclusivity periods.

Although generic drugs accounted for approximately 84 $\%$ of all prescriptions in 2013 [1], they remain controversial. Some physicians and patients resist using generic drugs and downplay their comparability to their brandname versions, despite no data supporting such perceptions $[2,3]$. Indeed, systematic reviews and well-controlled observational studies have consistently demonstrated the safety and effectiveness of generic drugs. Nonetheless, skepticism about generic drugs persists. It is highest among certain categories of drugs, including "narrow therapeutic index" drugs in which patients require precise doses to achieve clinical benefit without incurring toxicity $[4,5]$. Controversy related to generic drugs also flares in the context of generic drugs approved using product-specific determinations of therapeutic equivalence, in which the FDA approves a generic version using testing individualized to the physicochemical and pharmacokinetic characteristics of the drug at issue. Such product-specific testing may vary from standard therapeutic equivalence testing requirements and are usually challenged by the brand-name manufacturer, which contends that the product-specific testing does not properly ensure the safety and effectiveness of the generic product. We describe six generic drugs approved via product-specific regulatory pathways, and propose strategies for addressing skepticism that may emerge among patients and physicians by monitoring their safety and effectiveness following market authorization.

\section{The Use of Product-Specific Therapeutic Equivalence Standards}

Generic drug approval is premised on the notion that therapeutic equivalence ensures that generic drugs are as safe and effective as their brand-name counterparts. In a review of 12 years of bioequivalence data, the FDA found that the criteria used to evaluate generic drugs leads to the approval of generic versions that are bioequivalent to their brand counterparts within a narrow margin [6]. However, some drug products may have unique structural or functional attributes that necessitate product-specific approaches to therapeutic equivalence determinations. For example, if an in vivo pharmacokinetic study measuring the systemic distribution of the drug is unavailable because the drug acts locally on the gastrointestinal tract without being absorbed into the bloodstream, the FDA can instead permit in vivo tests that measure the acute pharmacologic effect of the active ingredient or a metabolite, or in vitro tests such as dissolution rate tests that reflect in vivo bioavailability [7].

On the one hand, regulatory flexibility to use productspecific pathways in the approval of generic drugs is critical to preserving the intent of the Hatch-Waxman Act: to ensure robust market competition among brand-name and generic drugs that can lead to lower prices for patients after expiration of market exclusivity. On the other hand, when certain tests are excluded from determinations of therapeutic equivalence or the FDA does not require additional pre-approval testing to confirm the effectiveness and safety of generic drugs approved using these product-specific approaches, it may also lead to the perception that the resulting generic drugs are less safe or less effective than their brand-name counterparts. Even when these perceptions are not warranted, they still must be addressed, as they could lead patients or physicians to avoid using generic alternatives. Moreover, in one cautionary recent episode, the FDA had to withdraw from the market the generic version of 300-mg extended-release bupropion, an antidepressant medication that had been approved with a bioequivalence waiver [8]. At the time of approval of the generic version in December 2006, the FDA waived bioequivalence testing of the 300-mg strength, choosing instead to allow the manufacturer to extrapolate data from bioequivalence tests of the $150-\mathrm{mg}$ strength because of concerns that the 300-mg strength might cause seizures in healthy volunteers. Following reports of lack of efficacy from patients receiving the generic 300-mg version after its approval, the FDA sponsored a study of the 300-mg strength in 24 healthy adult volunteers in 2012, which failed to demonstrate bioequivalence.

\section{Examples of Recent Generic Drug Approvals Using Modified Therapeutic Equivalence Standards}

The FDA has relied on product-specific pathways for determining therapeutic equivalence in two primary situations: (1) cases for which standard bioequivalence studies were not relevant, and (2) cases of complex molecules that may require specially tailored studies.

\subsection{Velafaxine Extended Release}

The first category includes the controversy around the drug venlafaxine extended release (Effexor XR, Wyeth), a 
serotonin and norepinephrine reuptake inhibitor antidepressant that is required in the label to be administered with food because taking it on an empty stomach causes nausea and vomiting. When the FDA issued a draft bioequivalence guidance, recommending only fed studies for subsequent generic market entrants because of the clinical risks of taking the drug on an empty stomach, Osmotica-the first generic manufacturer on the market—submitted a Citizen's Petition claiming that bioequivalence studies needed to be conducted in both the fed and fasting states, as usually is required for orally administered drug products to ensure that the safety and effectiveness of the generic versions were the same as with the brand-name version [9]. Ultimately, the FDA allowed subsequent approvals to be based on fed studies only [10].

\subsection{Acarbose and Vancomycin}

For the diabetic drug acarbose (Precose, Bayer) and the antibiotic vancomycin capsules (Vancocin, ViroPharma), the FDA was faced with drug products that act locally in the gastrointestinal tract without being systemically absorbed. In both of these cases, the FDA has permitted approval of generic manufacturers' products without the in vivo testing that would otherwise normally be required of generic manufacturers [11]. These decisions faced swift opposition. In the case of acarbose, the first generic manufacturer, Cobalt Pharmaceuticals, conducted both in vivo and in vitro studies, but after its approval, Cobalt submitted a Citizen's Petition to the FDA requesting other generic products also conduct in vivo bioequivalence testing, despite its low intestinal permeability. The FDA determined that it could accept scientifically valid in vitro studies alone for drugs that are not systemically absorbed, as long as pharmaceutical equivalence is demonstrated [11].

In the case of vancomycin oral capsules, the brand-name manufacturer ViroPharma filed a Citizen's Petition to seek reconsideration. The company argued that, while the drug acts locally in the gastrointestinal tract, a negligible amount is systemically absorbed and that, without in vivo bioequivalence studies, the FDA could not ensure that the rate and extent of absorption of the generic version was the same as that for the brand-name version. The company expressed concern that greater absorption would increase patients' risk of systemic toxicities. After a 2009 expert advisory committee endorsed the FDA's variant pathway, ViroPharma's petition was denied.

\subsection{Sodium Ferric Gluconate}

The FDA has also used product-specific therapeutic equivalence determination pathways in the cases of complicated molecules that could not be chemically classified as easily as small-molecule drugs. For example, sodium ferric gluconate (Ferrlecit, Watson) is an iron colloid made by a complex multistep process pioneered by Watson Pharmaceuticals over 5 decades ago [12]. In 2004, Watson filed a Citizen's Petition arguing that unless the process was reproduced exactly and the physicochemical characteristics identified as the same, clinical trials should be required. The company argued that physiochemical differences could have a negative impact on both the safety and efficacy of the generic product. The FDA agreed that the physicochemical properties of the active ingredient needed to be equivalent, but because of the reliability of the process of determining structural similarity, refused to mandate clinical trials [13].

\subsection{Salmon Calcitonin Nasal Spray}

Another example of a complex molecule is salmon calcitonin, which is a 32-amino acid polypeptide hormone involved in bone metabolism and is available in a nasal spray (Miacalcin NS, Novartis) for treating osteoporosis. When a generic manufacturer attempted to introduce a version of Miacalcin NS, Novartis argued in a Citizen's Petition that clinical studies demonstrating lack of immunogenicity and equivalent clinical outcomes should be required [14]. The FDA disagreed, finding that generic applicants only need demonstrate that the active polypeptide ingredient was the same because this simple peptide exhibited no significant secondary or tertiary folding to add to the complexity of the structure [15].

\subsection{Enoxaparin}

Similarly, enoxaparin (Lovenox, Sanofi-Aventis), an anticoagulant used to prevent or treat thromboembolic diseases, is a polysaccharide chain created from an unfractionated source heparin by chemical depolymerization. The brand-name manufacturing process produced a molecule that the manufacturer claimed had "yet to be fully characterized due to limitations on current analytical technology" [16]. In a 2003 Citizen's Petition, Aventis argued that generic manufacturers should be required to duplicate its unique process, or else complete full clinical trials; otherwise, they could not guarantee equivalent safety and effectiveness of the generic version. Instead, the FDA developed criteria for generic manufacturers to use to determine sameness between their products and brand-name enoxaparin, including the nature of the source material, laboratory measurements of anticoagulant activity, and the components of the product [17]. 
4 Assessing the Safety and Effectiveness of Generic Drugs Approved Using ProductSpecific Determinations of Therapeutic Equivalence in the Post-Approval Setting

As the FDA develops more product-specific pathways for drugs with unique structural properties, it will likely continue to face criticism over its choices about which sorts of tests to require and whether the tests ensure that generic products ultimately approved for marketing will be as safe and effective as brand-name versions. In the past, as exemplified by these six cases, this controversy generally has taken the form of claims made by brand-name manufacturers through the Citizen's Petition process that the methods that the FDA required were insufficient to assure therapeutic equivalence of the products. Certainly, some of the issues brought up in these Citizen's Petitions may have been frivolous and motivated by the financial interests of the brand-name manufacturers in maintaining their revenue by keeping generic competitors off the market. In each Citizen Petition, the manufacturers enumerated scientific rationales for their requests for greater testing, which the FDA reviewed in depth. Some of the petitions were denied in their entirety, others were partially accepted.

To shed additional light on these controversies and confirm whether the product-specific pathways that the FDA used in these six cases indeed ensured therapeutic equivalence, follow-up studies comparing patient experiences with the safety and effectiveness of the generic vs. brand versions of products approved could be conducted. While we have found no rigorous studies conducted of patient experiences with any of the six generic drugs described above, such studies are now beginning to be supported by the 2012 Generic Drug User Fee Act, which provided an influx of fees into the FDA associated with each application to market a new generic drug.

One mechanism for conducting such studies could be the FDA's Sentinel system. With electronic healthcare data covering 178 million individuals, Sentinel could facilitate large rapid assessments of the safety and effectiveness of new generic products [18]. The FDA is currently using the system to assess the safety of various drugs, vaccines, and blood products. Sentinel can accommodate both retrospective assessments as well as sequential assessments of newly approved products as data prospectively accrue in the system. These assessments directly inform FDA regulatory decisions and have led to several official drug safety communications, which are notices that the FDA disseminates about the safety of already-approved drugs [19].

The FDA could proactively use Sentinel to ensure the safety of generic drugs approved by modified regulatory pathways by comparing outcomes of patients who use generic drugs vs. outcomes of patients who use brand-name versions [20]. We led a recently published study that compared health outcomes of generic statin initiators to brand-name statin initiators, using some of the same methods that are available in Sentinel [21]. We found that patients initiating a low-cost, cholesterol-lowering drug had better medication adherence and, as a result, an $8 \%$ reduction in hospitalization for acute heart disease, stroke, and death compared with patients initiating a high-cost, cholesterol-lowering drug.

Large observational studies in electronic healthcare data can also be used to examine patient outcomes after switching between brand-name and generic versions of a drug [22] and to conduct time-series analyses of outcomes among patients using a particular drug when there is a large shift in use away from the use of one version, as commonly occurs after approval of a first generic version of a drug. These types of studies could also serve as models for assessing the safety and effectiveness of follow-on versions of biologic products in the post-approval setting. In March 2015, the FDA approved the very first follow-on biologic version of the white blood cell-stimulating agent filgrastim (Neupogen) called filgrastim-sndz (Zarxio), and rigorous post-market assessment of its safety and effectiveness will be key to the success of this pathway [23].

\section{Conclusion}

It is vital for the FDA to retain its flexibility to employ product-specific pathways to approve generic versions of some brand-name drugs, especially when certain studies (as in the case of venlafaxine) would put patients at unnecessary risk of harm. In addition, subsequently studying the clinical equivalence of brand-name and generic drugs is an important safeguard. Close monitoring of individual case reports will continue to be useful for identifying potential safety and effectiveness issues with generic drugs approved by product-specific therapeutic equivalence pathways. Active monitoring of these drugs products in large electronic healthcare databases should become another routine tool. Finally, required reporting to such registries as clinicaltrials.gov would increase transparency-currently, there is no requirement for public clinical trial registration of bioequivalence tests-and promote appropriate confidence of patients and physicians using these products.

\section{Compliance with ethical standards}

Funding Preparation of this manuscript was supported by a grant from the FDA Office of Generic Drugs to the Brigham and Women's Hospital. 
Conflicts of interest Aaron Kesselheim receives research funding from the Greenwall Faculty Scholar in Bioethics, and the Harvard Program in Therapeutic Science. Joshua Gagne is the Principal Investigator on research funding to the Brigham and Women's Hospital from the Harvard Catalyst, the FDA Mini-Sentinel program, the Patient-Centered Outcomes Research Institute, the Agency for Healthcare Research and Quality, and Novartis Pharmaceuticals Corporation. Members of the FDA Office of Generic Drugs provided comments on an early draft of this manuscript, but had no role in its preparation or approval.

\section{References}

1. Thomas, K. Brand-name drug prices rise sharply, report says. New York Times. November 29, 2012:B1.

2. Shrank WH, Cox ER, Fischer MA, Mehta J, Choudhry NK. Patients' perceptions of generic medications. Health Aff. 2009;28:546-56.

3. Shrank WH, Liberman JN, Fischer MA, Girdish C, Brennan TA, Choudhry NK. Physician perceptions about generic drugs. Ann Pharmacother. 2011;45:31-8.

4. Berg MJ, Gross RA, Haskins LS, Zingaro WM, Tomaszewski KJ. Generic substitution in the treatment of epilepsy: patient and physician perceptions. Epilepsy Behav. 2008;13:693-9.

5. Papsdorf TB, Ablah E, Ram S, Sadler T, Liow K. Patient perception of generic antiepileptic drugs in the Midwestern United States. Epilepsy Behav. 2009;14:150-3.

6. Davit BM, Nwakama PE, Buehler GJ, Conner DP, Haidar SH, Patel DT, Yang Y, Lu LX, Woodcock J. Comparing generic and innovator drugs: a review of 12 years of bioequivalence data from the United States Food and Drug Administration. Ann Pharmacother. 2009;43:1583-97.

7. Code of Federal Regulations. 21 CFR $\S 320.24 .2014$.

8. Woodcock J, Khan M, Yu LX. Withdrawal of generic budeprion for nonbioequivalence. N Engl J Med. 2012;367:2463-5.

9. Aikman, M. Re: ANDAs for venlafaxine $\mathrm{HCl}$ extended release tablets should include the results of bioequivalence studies conducted under both fed and fasting conditions. Re- Docket No. FDA-2010-P-0255. 20 May 2010. Available from: http://www. regulations.gov/\#!documentDetail;D=FDA-2010-P-0255-0001. Accessed 8 Mar 2015.

10. Woodcock J. Citizen's Petition response Re: Docket No. FDA2010-P-0255. 17 Nov 2010. Available from: http://www.regulati
ons.gov/\#!documentDetail;D=FDA-2010-P-0255-0013. Accessed 8 Mar 2015.

11. Food and Drug Administration. Draft guidance on Acarbose. Jul 2009. Available from: http://www.fda.gov/downloads/Drugs/ GuidanceComplianceRegulatoryInformation/Guidances/UCM170 242.pdf. Accessed 8 Mar 2015.

12. Novitch ME and Zuchero D. Citizen's Petition. Docket No.FDA2004-P-0494, 13 Feb 2004. Available from: http://www.regulati ons.gov/\#!documentDetail;D=FDA-2004-P-0494-0005. Accessed 8 Mar 2015.

13. Woodcock J. Citizen's Petition response Re: Docket No. FDA2004-P-0494. 31 Mar 2011. Available from: http://www.regulati ons.gov/\#!documentDetail;D=FDA-2004-P-0494-0008. Accessed 8 Mar 2015.

14. Rosen DL. Citizen's Petition. Docket No. FDA-2008-P-0616. 2 Sept 2005.

15. Woodcock J. Citizen's Petition response Re: Docket No. FDA200S-P-0367. 17 Nov 2008.

16. Bell L, Safir PO, Cunningham SL. Citizen's Petition. 19 Feb 2003. Available from: http://www.regulations.gov2003:26. Accessed 8 Mar 2015.

17. Throckmorton D. Citizen's Petition response Re: Docket No. FDA-2003-P-0273. 23 Jul 2010.

18. Platt R, Carnahan RM, Brown JS, Chrischilles E, Curtis LH, Hennessy S, Nelson JC, Racoosin JA, Robb M, Schneeweiss S, Toh S, Weiner MG. The U.S. Food and Drug Administration's Mini-Sentinel program: status and direction. Pharmacoepidemiol Drug Saf. 2012;21(Suppl. 1):1-8.

19. Moore TJ, Furberg CD. Electronic health data for postmarket surveillance: a vision not realized. Drug Saf. 2015 May 30. [Epub ahead of print].

20. Gagne JJ, Wang SV, Rassen JA, Schneeweiss S. A modular, prospective, semi-automated drug safety monitoring system for use in a distributed data environment. Pharmacoepidemiol Drug Saf. 2014;23:619-27.

21. Gagne JJ, Choudhry NK, Kesselheim AS, Polinski JM, Hutchins D, Matlin OS, Brennan TA, Avorn J, Shrank WH. Comparative effectiveness of generic and brand-name statins on patient outcomes: a cohort study. Ann Intern Med. 2014;161:400-7.

22. Gagne JJ, Avorn J, Shrank WH, Schneeweiss S. Refilling and switching of antiepildeptic drugs and seizure-related events. Clin Pharmacol Ther. 2010;88:347-53.

23. Sarpatwari A, Avorn J, Kesselheim AS. Progress and hurdles for follow-on biologics. N Engl J Med. 2015 May 6. [Epub ahead of print]. 